\title{
EXPERIENCES OF DESIGN AT HACKATHONS: INITIAL FINDINGS FROM AN INTERVIEW STUDY
}

\author{
Flus, Meagan; \\ Hurst, Ada \\ University of Waterloo
}

\begin{abstract}
Hackathons are short design events at which participants collaboratively progress through the entire design process. They pose opportunities for design research, but the existing research is limited, as is the understanding of design activity at hackathons. In our study, we summarize the hackathon design process of 10 interview participants from varying disciplines, levels of experience, and hackathon events. The summarized account reveals a decreased emphasis on the beginning phases of the design process, mainly problem definition, but an increased emphasis on the end, specifically the pitch portion of the event. These differences are mainly due to the limited time frame. We further assess the effect of time limitations at hackathons by comparing hackathons to other instances of design, emphasizing the impact of time constraints on iteration. We conclude our discussion with an exploration of the role expertise has on the design process by comparing the accounts of designers and developers.
\end{abstract}

Keywords: Hackathon, Design process, Human behaviour in design, Expertise, Collaborative design

Contact:

Flus, Meagan

University of Waterloo

Management Sciences

Canada

mhvflus@uwaterloo.ca 


\section{INTRODUCTION}

Hackathons, a popular phenomenon, are an untapped design research opportunity. Hackathon participants complete the entire design process in a limited time frame, usually $24-48$ hours. We view hackathons as short-term simulations of design in (near) real-life situations. The unique characteristics of hackathons, mainly the short time frame and limited resources, greatly condense design activity. Despite the research potential of hackathons, little is known about how participants design during the event (Flus and Hurst, 2021). Research on hackathons has the potential to expose ways in which the typical design process is challenged and adapted in this unique environment. In this paper, we present preliminary findings from an interview study that investigated how participants experience the design process at hackathons.

The rest of the paper is structured as follows. In section 2 we provide a background on the hackathon phenomenon, present highlights from a recently completed literature review on designing at hackathons, and articulate our research questions. In section 3 we describe our research method. Section 4 presents our findings. Finally, section 5 summarizes the main study conclusions and future research directions.

\section{BACKGROUND}

\subsection{Overview of the hackathon phenomenon}

There is no singular, agreed-upon definition of a hackathon (Komssi et al., 2015); however, by generalizing the characteristics of many hackathon events, it is easy to identify a pattern of characteristics and common variations. Hackathons are short design sprints where participants collaborate in small teams to ideate a solution to a complex and ill-structured problem (Briscoe and Mulligan, 2014). Problems that are chosen vary between hackathons, but are usually tech-centric (Briscoe and Mulligan, 2014) and align with the theme of the hackathon event, such as aircraft design (Saravi et al., 2018) or mental health (Birbeck et al., 2017). The aims of hackathon events also vary. Hackathons have been used to encourage ideation, develop solutions to problems within a company, and teach skills (most notably, design) (Flores et al., 2018).

Hackathons are very short events, usually spanning only 24-48 hours during which participants work continuously; however, sometimes the events are divided between days, weeks, months, or even years (De Oliveira et al., 2019; Hölttä-Otto et al., 2018; Richterich, 2019; Taylor et al., 2018; Truyen et al., 2016). Within the short time frame of the event, participants work quickly and continuously to "hack" together a solution, enlightening the origin of the name "hackathon" as a combination of "hack" and "marathon" (Briscoe and Mulligan, 2014).

Typically, the event goals, sponsors, schedule, and prizes are announced before registration for the event begins. The theme, challenges, and any design prompts may be provided before the event, or announced at its start. Some teams enter hackathons together, otherwise individuals partake in opportunities at the beginning of the hackathon that facilitate team formation. The event begins with a welcome presentation, and over the remainder of the event, teams must identify a problem and build the solution. At the end of the event, participants present their projects in a pitch competition and compete for prizes.

Since the first known use of the term "hackathon" to describe an event in 1999 (Briscoe and Mulligan, 2014), hackathons have quickly gained popularity (Artiles and Wallace, 2013). There were 5,636 hackathons globally in 2018 (hackathon.com, 2018). Hackathons tend to mainly attract participants in Science, Technology, Engineering, or Mathematics (STEM) fields; however, there is growing interest in other disciplines, such as design and business.

\subsection{Designing at hackathons}

While hackathons are a rich and authentic environment in which people design, the activity of designing in this setting has not yet been comprehensively studied. In our recently completed literature review on design activity at hackathons (Flus and Hurst, 2021), we gained some insight on (1) the design process participants tend to follow during a hackathon, (2) the ways in which hackathon organizers can encourage a design process by how they structure a hackathon event, and (3) the pedagogical potential of hackathons for design teaching and learning. The main conclusion of our review was that while hackathons are a popular setting in which people design, studies of design cognition and behaviour at hackathons are limited, and thus, our understanding of how design transpires at hackathons is fragmented and incomplete. 
Our review presented a number of open questions and future research directions, which we briefly summarize here. First, due to the nature of the hackathon format, hackathon participants design continuously throughout the event duration, taking few if any breaks. They are therefore severely deprived of the natural incubation periods (Woodworth, 1938), which have been shown to reduce design fixation and improve idea generation (Cardoso and Badke-Schaub, 2009; Tsenn et al., 2014). Therefore, a question that emerged was how the hackathon structure was impacting the availability of these incubation periods and the quality of the eventually produced designs.

Second, it appears that two "opposing" forces operate at hackathons. On one hand, the extreme time pressure at the hackathon would point at an increase in "fast designing" (Kannengiesser and Gero, 2019), a tendency for designers to quickly transition from requirements to readily available designs (produced from past experience). On the other hand, the hackathon setting places the designer in a state of increased uncertainty (Ball and Christensen, 2019), which would require more careful and deliberate - or "slow" - designing. We therefore identified the need to understand how designers overcome high levels of uncertainty quickly during hackathons (Flus and Hurst, 2021).

Third, participants' activity at a hackathon is also influenced by their own characteristics, including area and extent of expertise, previous experiences, and past participation in hackathons. The influence of these factors is combined in further unpredictable ways as participants come together in teams, often in an ad-hoc manner.

\subsection{Study aims}

The focus on this paper is on this third topic - the role that participants' individual characteristics play in how they experience the design process at hackathons. As such, we articulated two research questions:

1. How are the various stages of the design process experienced at hackathons?

2. How does that experience vary depending on the participants' prior experience and expertise in design?

\section{METHODOLOGY}

This research employed semi-structured interviews. To address the research gaps identified in the literature review, mainly the limited number of study participants from only a handful of events, we opened our recruitment to any person who had participated in at least one hackathon, taking a "big net" approach to learning about experiences at different hackathons from participants in different disciplines. Participants were recruited via social media posts and networking channels, as well as through snowball sampling. In total, 10 semi-structured interviews, each 45 to 60 minutes in length, were conducted remotely in spring to fall 2020 . The first author led all interviews, while the second author was present (but not an active participant) in all but three of the interviews. All participants were located in Canada and the United States. Interviews are still on-going as part of a larger study.

Interviews were structured as follows. The interviews began with a set of general demographic and background questions related to the participants' domain of expertise and experience participating in hackathons, including the number of hackathons previously attended, their motivations for attending, and their role at them. Participants were then asked questions about the activity of design at hackathons, beginning with the question, "define 'design' as you understand it" before asking them to describe their design process at hackathons and compare the experience to other, more typical, design projects.

Table 1 presents a comprehensive overview of the ten study participants. Nine presented as female, and the remaining participant presented as male. The participants' highest level of completed or inprogress education was an undergraduate degree. They had attended an average of 4.8 hackathons, varying in their motivations, with reasons such as to win, to network, and to learn being the most frequently stated. Participants had an average of approximately 2 years of professional experience; of the 10 participants, six self-identified as developers (labelled Dev\#) and four as designers (Des\#).

All interviews were recorded and transcribed. The first author, who had participated in all interviews and could engage in a holistic reflection on the participants' responses, then conducted a thematic analysis (Braun and Clarke, 2006) on the transcripts. The themes identified belong to two main topics. 
Table 1. Summary of study participants' hackathon experience

\begin{tabular}{|c|c|c|c|c|c|}
\hline ID & $\begin{array}{l}\# \text { of } \\
\text { hack- } \\
\text { athons }\end{array}$ & $\begin{array}{l}\text { Role at } \\
\text { hackathon }\end{array}$ & $\begin{array}{l}\text { Motivation to attend } \\
\text { hackathon(s) }\end{array}$ & $\begin{array}{l}\text { Work } \\
\text { experience } \\
\text { (years) }\end{array}$ & $\begin{array}{l}\text { Focus of } \\
\text { attended } \\
\text { hackathons }\end{array}$ \\
\hline Des1 & 3 & $\begin{array}{l}\text { UX } \\
\text { Designer }\end{array}$ & $\begin{array}{l}\text { To challenge self, to have fun, } \\
\text { to win }\end{array}$ & $7-8$ & $\begin{array}{l}\text { Healthcare, arts, } \\
\text { company-internal }\end{array}$ \\
\hline Des2 & 4 & $\begin{array}{l}\mathrm{UX} \\
\text { Designer }\end{array}$ & To learn, to win & $6+$ & $\begin{array}{l}\text { Various } \\
\text { (healthcare, social } \\
\text { impact, tech) }\end{array}$ \\
\hline Des3 & 13 & $\begin{array}{l}\text { UX/UI } \\
\text { Designer }\end{array}$ & $\begin{array}{l}\text { For fun, to learn, networking, } \\
\text { to win }\end{array}$ & $0-1$ & $\begin{array}{l}\text { Various } \\
\text { (blockchains, } \\
\text { mental health, } \\
\text { product } \\
\text { development) }\end{array}$ \\
\hline Des4 & 1 & $\begin{array}{l}\text { UX } \\
\text { Designer }\end{array}$ & Networking & $2+$ & Healthcare \\
\hline Dev1 & 8 & Developer & To win & $1-2$ & $\begin{array}{l}\text { Software } \\
\text { development }\end{array}$ \\
\hline Dev2 & 1 & Developer & For the experience, networking & $0-1$ & Social change \\
\hline Dev3 & 2 & Developer & To learn & $1-2$ & $\begin{array}{l}\text { Tech-centric and } \\
\text { company-internal }\end{array}$ \\
\hline Dev4 & 2 & $\begin{array}{l}\text { Developer } \\
\text { (business) }\end{array}$ & $\begin{array}{l}\text { To learn about hackathons to } \\
\text { help with organizing one }\end{array}$ & $0-1$ & $\begin{array}{l}\text { Civic hackathon } \\
\text { (student focussed) }\end{array}$ \\
\hline Dev5 & 8 & $\begin{array}{l}\text { Front-end } \\
\text { developer }\end{array}$ & To learn & $0-1$ & $\begin{array}{l}\text { Tech-centric, } \\
\text { virtual }\end{array}$ \\
\hline Dev6 & 6 & $\begin{array}{l}\text { UX } \\
\text { developer }\end{array}$ & $\begin{array}{l}\text { Free food/swag, networking, } \\
\text { build resume }\end{array}$ & $0-1$ & $\begin{array}{l}\text { Tech-centric, } \\
\text { education, } \\
\text { healthcare }\end{array}$ \\
\hline
\end{tabular}

The first related to the type of activity described and its place in the design process, for example team formation, problem finding, problem defining, planning, building, and pitching. Also included in this topic is unique characteristics of designing at hackathons, particularly the nature of iterations at hackathons. The second topic related to the role of expertise at hackathons.

\section{FINDINGS}

\subsection{The design process at hackathons}

Our synthesis of the literature in Flus and Hurst (2021) led us to propose that design at hackathons follows what we termed an "altered" Double Diamond Design Process (Figure 1). We argued that the steps participants appear to follow during hackathons roughly align with the Discover-DefineDevelop-Deliver stages of the Double Diamond Design Process (The Design Council, 2005); however, three main differences emerge: (1) increased emphasis on team formation, (2) decreased emphasis on problem definition, and (3) increased emphasis on delivery.

In what follows, we present a summary of the hackathon design processes as described by our study participants, organized according to the phases of the altered Double Diamond Design Process proposed in Flus and Hurst (2021).

\subsubsection{Discover}

The first stage, Discover, involves the exploration of the problem space. At hackathons, participants work in teams; as such team formation is a critical activity. The interviews revealed different methods by which teams form. While some participants attended the hackathon with a pre-established team (Des1, Des3), sometimes these teams fell through (Des2). The most commonly reported method 
of team formation was creating teams while at the hackathon, typically by sitting down at a table with strangers and asking to join their team (Des2), posting on the hackathon's communication platform (i.e., Slack) (Dev5), or via idea pitches (Des4).

The team formation step is critical to the functioning of the hackathon team, in part because team composition sets constraints on what the team can accomplish. Des3 outlined this process as follows:

"We kind of communicate and see what our strengths are so we know like kind of the limits of what we can build. So, for example, if someone on the team is like really good at AI [artificial intelligence], then will be like maybe we can incorporate like machine learning into our project" (Des3).

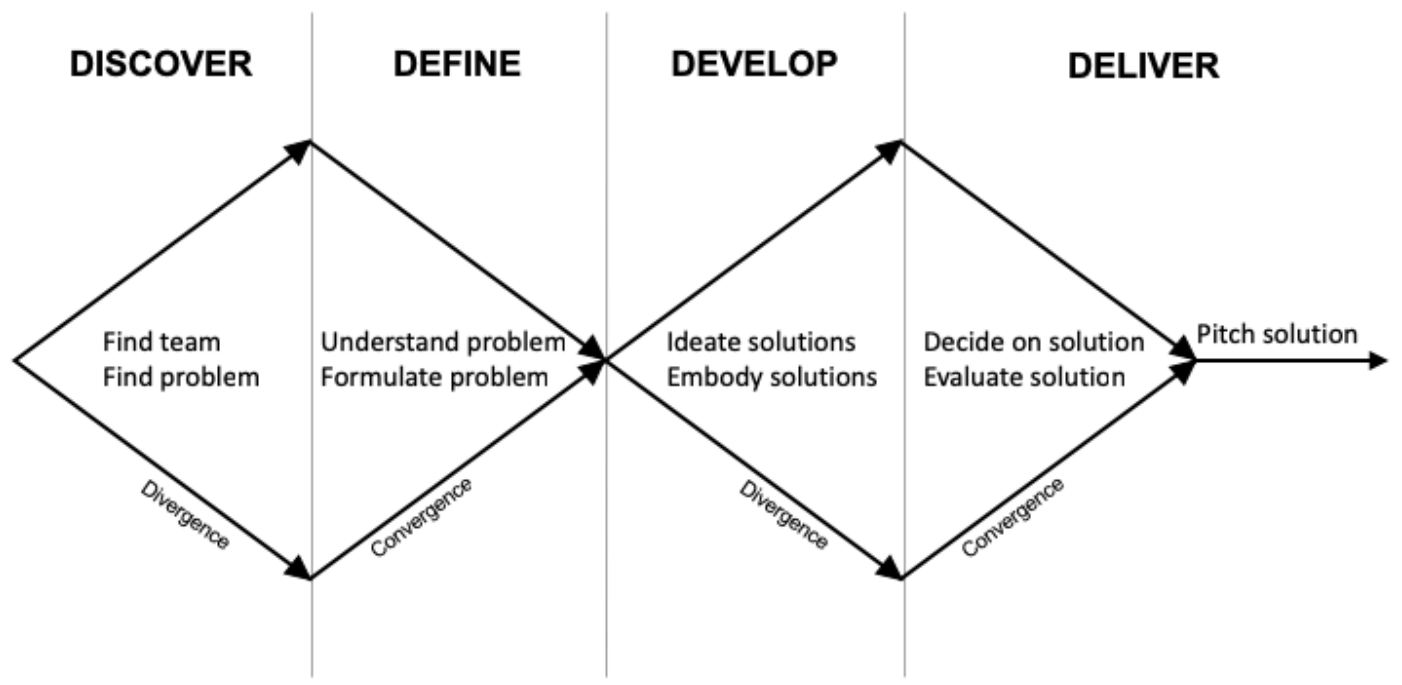

Figure 1. The altered double diamond process (Flus and Hurst, 2021).

Not surprisingly, many study participants mentioned intentionally forming teams based on skill set. For example, Dev5, a developer with knowledge on front-end development, searched for teams that needed a front-end developer, or sought back-end developers to join their team. Beyond subject matter knowledge, as teams form, members also discuss interests, their motivations for attending the event (i.e., if they want to win, learn, or network), and their expectations for group behaviour (i.e., if they will work through the night or not).

Once teams are established, they begin the process of finding a problem. As mentioned previously, this may occur simultaneously with team formation, such that teams are formed on the basis of a problem idea and/or shared interests. In the event that teams are formed without an idea for a problem, teams may look to the theme (e.g., "encouraging connecting in an aging population") or "tracks"/"categories" (e.g., "business") of the hackathon for inspiration (Des 2, Des4, Dev4, Dev5). Teams may look to these as prompts, especially since many of the tracks originate from sponsors, and thus have prizes attached to them (Dev5). Other methods for problem finding include researching topics that may be of interest by gathering more information on the problem and existing solutions (Dev4), and both individual and group brainstorming (Dev4, Des4).

\subsubsection{Define}

In the next phase of the framework, Define, designers must narrow the problem space through the refinement of requirements and constraints. At hackathons, defining the problem is heavily constrained by the very limited time available for teams to complete their designs. Des4, who explained the most thorough process of understanding and formulating the problem at hackathons, led their group through a series of user experience activities, mainly persona development and journey mapping. The stated purpose of this was to better understand the user for whom they were designing, identify the key touchpoints their solution had to address, and establish a set of requirements. Similarly, Des2 emphasised designing with a user in mind. They recalled expressing concern for their team's idea because it was not informed by a true user. In an attempt to identify requirements, they asked questions to the developers in their team including, "What benefit is it really providing?" and "Who's providing the data and why would they use this?". They also asked questions to subject matter experts (due to the lack of users at the event) to ensure their idea would address a real need. Des2 
highlighted the importance of checking assumptions and establishing a strong foundation on which a solution is developed.

Interviews revealed a de-emphasis of research at hackathons. Only designer participants revealed an attempt to thoroughly understand their problem prior to beginning development. Many designers reported that developers in their teams sought to begin building promptly after choosing a project topic.

Many study participants mentioned creating a project development plan with their team members as part of the problem formulation phase. This process typically began with identifying the must-have features their solution should include (Des3). Team members would then delegate tasks that aligned with the role team members took in the team (Dev4, Des4), roughly identifying roles as front-end and back-end developers, designer, and in some cases, a business lead.

\subsubsection{Develop}

In the third phase, Develop, participants begin the process of building the solution. This stage is typically marked by solution ideation and prototype building activities. Our study participants described how progress at this stage is achieved by delegating tasks that occur simultaneously. The building of the solution happens in consecutive collaboration. For example, Des3 outlined the building phase as beginning with designing wireframes to inform the engineers on the structural interface, which allows them to begin coding. Then interface design decisions, such as colour and font, are decided, which allows the development of the front-end. Interview participants also mentioned the use of sketching to establish designs (Dev4) and the importance of GitHub for storing their code and effectively collaborating (Dev6). Des2 eloquently explained this phase as "an iterative loop of asking: how far have we gotten? How's it going? Did we do the thing that we intended to do? What's the next step we need to do?". Few study participants mentioned testing their prototypes, but Des2 described revisiting requirements to ensure the solution is appropriate and Dev6 spoke to how the team would check each other's work, ask for feedback, and help solve issues individual team members could not solve on their own.

\subsubsection{Deliver}

In the fourth and final phase, Deliver, designers evaluate and finalize their solution. The altered Double Diamond process extends this phase to emphasize the pitch portion of hackathons (Flus and Hurst, 2021), represented as an extra arrow in Figure 1 to accurately represent the significant time and resources dedicated to this activity. Hackathons are defined by competition, with top placements often awarded financial prizes from corporate sponsors. As a result, participants dedicate significant effort to developing and practicing their pitch. Hackathons often conclude with a presentation of solutions in front of all participants and an announcement of winners.

Accordingly, the final activity described by the study participants was the final pitch - usually comprising of a slide deck (Des2) and demonstration (Dev5) - to a set of judges, which often includes sponsors, who score the projects and award final prizes. Depending on the hackathon, there may be opportunities to practice the pitch in front of judges before the final competition (Des2). Some teams place more emphasis on pitching than others. Most study participants stated that the product pitched at hackathons does not have to be finished; instead they presented a partial prototype, sketches, and outline of their intentions (Des3) in order to demonstrate the potential of their project. Dev1 compared the pitch to a magic show, explaining that "the pitch is kind of like performing a slight of hand trick under the table", meaning that as long as the solution appears to work, it does not actually have to work.

\subsection{Prioritization of the design phases}

Due to the notable time constraint at hackathons, it became clear that participants were not able to thoroughly engage with the entire design process, necessitating prioritization of some design phases over others. We propose that the nature of this prioritization of design phases is related to the participant's role at the hackathon (i.e., designer or developer) and their motivation for the attending the event.

In general, participants broadly identified three main phases of activity at hackathons: problem finding and formulation, solution building, and pitching. When asked to reflect on which phase teams spent the most amount of time, Des2 claimed, "I think that the building does have to take the most amount of time because there are elements of it you just can't speed up anymore". Many participants agreed that 
the building phase of the hackathon was the longest, but there was disagreement on which of the three phases is most important.

Two participants claimed the first portion of the hackathon is most important. Des4 emphasized the importance of requirement gathering, identifying constraints, and defining the functions of the artefact because this exploratory work is necessary to validate the remainder of the design process. They explained that "once we had a very clear kind of concept of what we were doing, the [building phase] was actually quite straightforward". Dev4 agreed with Des4 that the beginning phase of the hackathon is the most important, emphasizing the necessity of establishing a healthy rapport with the team.

Des2 offered a different opinion, claiming that the building portion was both the longest and most important. Other participants also expressed a similar opinion, explaining that without a welldeveloped product, the judging will not end favourably. That being said, Des2 also added that the efforts given to developing the idea and checking assumptions needs to be great in order for the building to progress smoothly.

Dev1 argued that the pitch is the most important in order to win. They stated that if the idea is pitched well, regardless of whether the team has a working model, the chances of winning are greater. Des 3 expressed a similar sentiment, adding that judges "aren't necessarily like digging into your code and like trying to figure out if it works or not"; so, efforts are given to presenting the solution over making it work.

Dev5 offered an interesting insight into the connection between the phase of the design process that is given more effort and the motivation for attending the event. They stated that, "the most important part for winning I guess is using or like creating something that looks good and also that has a cool feature". In contrast, they said if attending the hackathon to learn, defining the problem is most important as it is during the beginning phases when it is decided what the focus will be; thus, the topic of the project should be reflective of the learning objective. This contribution suggests the importance of the team formation phase in that teams must align their goals of the event in order to give most effort to the parts of the project that will best achieve those goals. It also offers an explanation for why only designers were found to complete research, where developers wished to start building immediately since these two roles may have different goals. This point will be further explored in Section 4.7.

\subsection{The effect of time limitations at hackathons}

While hackathons centre on design activity, the nature of the hackathon events affect the resulting design process, such that it differs from more traditional design projects. In Flus and Hurst (2021) we identify a number of such factors, including the stress and fatigue resulting from the condensed time frame. Our interviews with hackathon participants provide some interesting insights about the unique ways in which the hackathon format affects how people design at these events.

\subsubsection{How hackathons differ from other design}

The extreme time pressure during hackathons usually results in strategic use of resources to ensure a team succeeds. In comparison to other design projects, our interviews revealed an increased emphasis on pitching and a decreased dedication to conducting extensive research prior to building. Only two study participants, Des2 and Des4 used user-centred approaches in their hackathon design approaches. Both participants expressed how challenging it was to convince their team members on the importance of these practices; yet, both teams received prizes for the most intentional and researched projects, demonstrating how emphasizing a deliberate problem defining phase during a hackathon can be advantageous to the team. Des2 described the difference between hackathons and previous design experiences as follows: "In a hackathon, like it's really hard to get people to like pause and like actually plan because you've got so little time to do a lot of it. I think in comparison, in a design project you spend like so much time scoping what you're doing and validating that it's a problem or like investigating the system that it's going to fit in and how that's all going to link together" (Des2). Dev6 also stated that the main difference between hackathons and other design experiences is the limited ability to speak with the intended users of solution being designed. Similarly, Des 3 described how they understood the importance of conducting research and tests when designing, but did not encourage these practices during hackathons because there was not sufficient time.

Des4 expressed that hackathons differ in how team members must collaborate. In other design projects there is more time for dependent tasks to happen consecutively. For example, traditional design 
collaborations may involve a thorough investigation that informs the development of requirements, after which development starts. In hackathons, however, "you can't have your software developers sitting around doing nothing for the first 2 days and then have on Thursday to like kick into gear and start to do something" (Des4). This study participant claimed their team would not be finished a task in its entirety before it had to be passed to the next member. Due to the short time frame of the event, it is more efficient to work based on the information available at the time and adjust as necessary.

\subsubsection{Design iteration at hackathons}

Iteration, a critical practice in design, is the repetition of design tasks (Smith and Eppinger, 1997), often with the goal of improving the artefact based on new information about the problem as it is learned. It is understood that iteration occurs not only within a design phase (e.g., iterations of prototypes in the Develop phase), but also between design phases (e.g., revisiting the Define phase to re-evaluate the problem definition after attempting prototypes in the Develop phase). In our study, we found that participants had divergent opinions on the extent to which design iterations occur at hackathons.

Iteration is central to agile design methods, and the connection between hackathons and agile methods was evident in many interviews. Des2 related the rounds of iteration within their team to agile design sprints, and outlined them as rounds of making an assumption, validating it, building, and revisiting the assumption to ensure their solution solved the problem appropriately. Dev5 was familiar with agile methods, such as stand-ups, and discussed their team's tendency to reconvene when they reached certain "milestones" such as when they finished larger tasks. Dev6 explicitly described their team's strategy as beginning each day with a discussion of outstanding tasks and developing a plan to finish them. Despite hackathons and agile methods sharing many features, mainly trying to progress through the design process quickly, Dev5 reported that other (non-hackathon) design experiences afforded more time to develop and implement agile practices.

For both Des2 and Des4, iteration was a fundamental and seemingly built-in practice to their hackathon process, which they were able to identify and speak to in-depth. This was not the case for all participants. Dev6 claimed that "there's like almost no iterative process" due to the time constraint and they "wonder if the [hackathon design] process is just one round of a product development" rather than many cycles of the Double Diamond Process, as is typical in other design projects. In other words, the design process is whole, but singular.

There was also a third view on design iterations, a middle ground approach more aligned with the practice of iteration within a phase. Des3 claimed that the iteration which occurs in their hackathon design process must be quick and independent. They reported producing many sketches of a design and quickly iterating on them before making a final decision. Once they handed off their designs to the engineers, "it's not worth the effort to change it because they are already starting to develop it" (Des3). In this case, once a decision was made, it could not be changed.

The typical design process is very iterative, so the claim that none occurred during hackathons, or if it did was very small, was concerning. Upon reflecting on these discussions, we noticed an interesting pattern. The study participants who self-identified as designers concluded that iteration, to some degree, occurs at hackathons. In contrast, self-identified developers did not. We then hypothesize that iteration does occur at hackathons, but the ability to identify the practice and critically discuss it, is limited to those with an understanding of and experience in design.

\subsection{The role of expertise at hackathons}

In Flus and Hurst (2021) we hypothesized that expertise - in design, another subject domain, and hackathons themselves - affects how a hackathon participant designs at hackathons.

An individual can have different levels of expertise in different skills (Dreyfus and Dreyfus, 1980); for example, a designer can be an expert in product design but a novice in web design. When asked if it is advantageous to have a diversity of subject matter expertise in a hackathon group, Dev4 said that diversity of expertise is important because, "members from different backgrounds can bring different skills and experiences to the table... If there are team mates with different expertise, it is easier to delegate tasks and have all the tools necessary to finish the project" (Dev4). Interview participants self-identified as either a developer or designer, indicating two major roles in hackathon teams. As we saw in team formation, participants sought out diverse teams with groups tending to be comprised of 
participants with diverse education, experience, and roles, demonstrating that hackathons typically enable collaboration between different topic experts (Frey and Luks, 2016).

This finding raises the question of the role participants who have interdisciplinary knowledge play in hackathon teams. That is, what if a participant is both a developer and a designer? Our interviews revealed that study participants who have expertise in both development and design, for example Dev6, would take on a role at the hackathon that aligned with the subject matter in which they had the highest expertise. For Dev6, this was software development.

Another interesting insight we gained from our interviews was with regard to the specific role of designers in hackathon teams. More experienced designers can rely on stored solutions accessible from their repertoires (Schön, 1988), which they can apply to new design problems. They can thus quickly transform requirements into design solutions (Kannengiesser and Gero, 2019), an ability that is crucial in high-pressure settings such as hackathons. It can be inferred that experienced designers are better equipped to succeed in a hackathon because they can more easily rely on their past experience. Our research found that the presence of a designer in a hackathon team resulted in more advanced research. Dev6 has some design knowledge, so was able to discuss the addition of a designer in a team with more pointed language. They stated that, "when there is a designer [in a hackathon team], there's added precautions. There are like building blocks to kind of prevent guesswork from happening", implying that a designer often forces the team to think about the problem more holistically.

Dev6 presented an example from when their team developed a software in which the user typed a command. They said that the designer caught the additional detail that the command had to be typed using only English letters. This prevented the case of judges typing in a number and receiving an error. In this example, the presence of the designer on the team filled understanding gaps and checked assumptions.

Based on these findings, we hypothesize that a designer in a hackathon team plays a valuable role such that the design process is more intentional. This conclusion requires further exploration on how prior design knowledge and experience impacts design behaviour at a hackathon and is the focus of our ongoing interview study.

\section{CONCLUSIONS AND FUTURE WORK}

Hackathons are popular design-centred events that pose new opportunities for design research. A set of 10 interviews with designers and developers who had previously participated in hackathons provided an account of diverse design experiences at hackathons with the aim of providing insight on the characteristics of design activity in this unique setting. A thematic analysis of the interview responses provided further support for the "altered" Double Diamond Design Process that had been previously proposed in Flus and Hurst (2021). Specifically, while hackathon participants follow a design process that resembles the Double Diamond Design Process (The Design Council, 2005), there are some unique differences related to the disproportionate time, resources, and importance that are taken by two activities: team formation and preparing for and delivering the final pitch. Further, the short time frame restricts the participants' ability to conduct thorough research, forces increased interdependence between team members, and limits design iteration. Finally, participants experience and perceive the design process at hackathons differently, depending on their area of expertise, knowledge and experience with design, and participation in previous hackathons.

The findings from this round of interviews have been very valuable. The emerging themes of the effects of a time limit and expertise on the design process offer promising opportunities for further research. We will continue to explore design experiences at hackathons as we conduct more interviews with an increasingly diverse participant pool. The objective of this continuing work is to further dissect the nature of collaborations between designers and developers. We hope this research will reveal patterns associated with expertise and provide insight on team dynamics at hackathons.

\section{REFERENCES}

Artiles, J.A., Wallace, D.R. (2013), "Borrowing From Hackathons: Overnight Designathons as a Template for Creative Idea Hubs in the Space of Hands-On Learning, Digital Learning, and Systems Re-Thinking”.

Ball, L.J., Christensen, B.T. (2019), “Advancing an understanding of design cognition and design metacognition: Progress and prospects”, Design Studies, Vol. 65, pp.35-59. https://doi.org/10.1016/j.destud.2019.10.003 
Birbeck, N., Lawson, S., Morrissey, K., Rapley, T., Olivier, P. (2017), "Self harmony: Rethinking hackathons to design and critique digital technologies for those affected by self-harm", Conference on Human Factors in Computing Systems, pp. 146-157. https://doi.org/10.1145/3025453.3025931

Braun, V., Clarke, V. (2006), "Using thematic analysis in psychology", Qualitative Research in Psychology, Vol. 3, No. 2, pp. 77-101. https://doi.org/10.1191/1478088706qp063oa

Briscoe, G., Mulligan, C. (2014), "Digital Innovation: The Hackathon Phenomenon".

Cardoso, C., Badke-Schaub, P. (2009), "Give Design a Break? The Role of Incubation Periods During Idea Generation”, International Conference on Engineering Design. ICED'09, Stanford, USA.

De Oliveira, C.M.C., Canedo, E.D., Faria, H., Amaral, L.H.V., Bonifacio, R. (2019), “Improving Student's Learning and Cooperation Skills Using Coding Dojos (In the Wild!)", Frontiers in Education Conference, FIE. https://doi.org/10.1109/FIE.2018.8659056

Dreyfus, S.E., Dreyfus, H.L. (1980), A Five-Stage Model of the Mental Activities Involved in Directed Skill Acquisition: Defense Technical Information Center, Fort Belvoir, VA. https://doi.org/10.21236/ADA084551

Flores, M., Golob, M., Maklin, D., Herrera, M., Tucci, C., Al-Ashaab, A., Williams, L., Encinas, A., Martinez, V., Zaki, M., Sosa, L., Pineda, K.F. (2018), "How can hackathons accelerate corporate innovation?”, Advances in Production Management Systems, Production Management for Data-Driven, Intelligent, Collaborative, and Sustainable Manufacturing. APMS 2018. Springer, Cham, pp. 167-175. https://doi.org/10.1007/978-3-319-99704-9_21

Flus, M. and Hurst, A. (2021) "Design at hackathons: new opportunities for design research,” Design Science. Cambridge University Press, 7, e4. https://dx.doi.org/10.1017/dsj.2021.1.

Frey, F.J., Luks, M. (2016), The innovation-driven Hackathon - One means for accelerating innovation, in: ACM International Conference Proceeding Series. https://doi.org/10.1145/3011784.3011794

Hackathon.com. (2018), [online]. Available at: https://www.hackathon.com:443/ (accessed 10.7.20).

Hölttä-Otto, K., Niutanen, V., Eppinger, S., Browning, T.R., Stowe, H.M., Lampinen, R., Rahardjo, A. (2018), "Design sprint for complex system architecture analysis", ASME Design Engineering Technical Conference. https://doi.org/10.1115/DETC2018-85774

Kannengiesser, U., Gero, J.S. (2019), "Design thinking, fast and slow: A framework for Kahneman's dualsystem theory in design", Design Science, Vol. 5, No. 10. https://doi.org/10.1017/dsj.2019.9

Komssi, M., Pichlis, D., Raatikainen, M., Kindstrom, K., Jarvinen, J. (2015), "What are Hackathons for?”, IEEE Software, Vol. 32, pp. 60-67. https://doi.org/10.1109/MS.2014.78

Richterich, A. (2019), "Hacking events: Project development practices and technology use at hackathons", Convergence, Vol. 25, pp. 1000-1026. https://doi.org/10.1177/1354856517709405

Saravi, S., Joannou, D., Kalawsky, R.S., King, M.R.N., Marr, I., Hall, M., Wright, P.C.J., Ravindranath, R., Hill, A. (2018), "A systems engineering hackathon - A methodology involving multiple stakeholders to progress conceptual design of a complex engineered product”, IEEE Access 6, pp. 38399-38410. https://doi.org/10.1109/ACCESS.2018.2851384

Schön, D.A., 1988. Designing: Rules, types and worlds. Design Studies 9, 181-190.

Smith, R.P., Eppinger, S.D. (1997), “A Predictive Model of Sequential Iteration in Engineering Design”, Management Science, Vol. 43, pp. 1104-1120.

Taylor, N., Clarke, L., Skelly, M., Nevay, S. (2018), "Strategies for engaging communities in creating physical civic technologies", Conference on Human Factors in Computing Systems, https://doi.org/10.1145/3173574.3174081

The Design Council. (2005), "The "Double Diamond" Design Process".

Truyen, F., Taes, S., Colangelo, C., Wyns, R. (2016), “Getting Creative With Europeana: Innovative Strategies \& New Tools For Education”, Edulearn16: 8th International Conference On Education And New Learning Technologies, Edulearn pp. 6606-6614.

Tsenn, J., Atilola, O., McAdams, D.A., Linsey, J.S. (2014), “The effects of time and incubation on design concept generation", Design Studies, Vol. 35, pp. 500-526. https://doi.org/10.1016/j.destud.2014.02.003

Woodworth, R.S. (1938), "Experimental psychology". Holt. 